\title{
Human Face Detection and Tracking for Age Rank, Weight and Gender Estimation based on Face Images using Raspberry Pi Processor
}

\author{
Mr. Sarang C. Zamwar, Dr. S. A. Ladhake, Mr. U. S. Ghate \\ (PG Scholar [Digital Electronics], Department of Electronics and Telecommunication, Sipna College of \\ engineering and technology, Sant Gadge Baba Amravati University, Amravati (M.S.), India \\ (Principal, Sipna College of engineering and technology, Amravati (M.S.), India) \\ (Assistant Professor, Department of Electronics and Telecommunication Sipna College of engineering and \\ technology, Amravati (M.S.), India)
}

\begin{abstract}
This paper describes the technique for real time human face detection and tracking for age rank, weight and gender estimation. Face detection is involved with finding whether there are any faces in a given image and if there are any faces present, track the face and returns the face region with features of each face. Here it describes a simple and convenient hardware implementation of face detection method using Raspberry Pi Processor, which itself is a minicomputer of a credit card size. This paper presents a cost-sensitive ordinal hyperplanes ranking algorithm for human age evaluation based on face images. Two main components for building an efficient age estimator are facial feature extraction and estimator learning. Using feature extraction and comparing with our input database in which we have different age group face images with weight is specified according to that we also specify weight category i.e. under weight, normal weight and overweight . In this article we present gender estimation technique, which effectively integrates the head as well as mouth motion information with facial appearance by taking advantage of a unified probabilistic framework. Facial appearance as well as head and mouth motion possess a potentially relevant discriminatory power, and that the integration of different sources of biometric data from video sequences is the key approach to develop more precise and reliable realization systems.
\end{abstract}

Keywords - Age rank, human face detection, Ordinal hyperplanes ranking, Raspberry pi Processor, Tracking, Unified probabilistic framework, Weight.

\section{INTRODUCTION}

Most face detection algorithms are designed in the software field and have a high detection rate, but they often need several seconds to detect faces in a single image, a processing speed that is insufficient for real-time applications. A simple and easy hardware implementation of face detection system using Raspberry Pi 3 Model B Processor, which itself is a minicomputer of a credit card size and is of a very low price.

Automatic age estimation, which involves evaluating a person's exact age or age-group and weight estimation, is a important topic in human face image understanding. A effective gender classification procedure can improve the performance of many different applications, including person recognition and smart humancomputer interfaces. Here use of Raspberry Pi board as a platform for this procedure. Camera $\mathrm{Pi}$ is an excellent add-on for Raspberry Pi 3 Model B Processor, to take pictures and record quality videos, with the possibility to apply a considerable range of configurations and effects. For real time and from specific image face recognition, i.e. Object detection, is done and the proposed system is tested across various face databases, with and without noise and blurring effects. Efficiency of the system is examine by calculating the Face detection rate for each of the database. The results reveal that the proposed system can be used for face recognition even from low quality image and shows outstanding performance efficiency. Automatic age estimation, which involves evaluating a person's exact age or age-group, is a important topic in human face image understanding. The task of estimating exact human age adopts a dense representation of the age labels (e.g., from 0 to 80), and the task of age-group estimation divides the labels only into rough groups (e.g., elder, adult, and teenage/children). In this paper, we focus on the setting of the former task that can be applicable to more general situations. Nevertheless, the proposed technique can be used for age-group estimation as well. Two main components for building an efficient age estimator are facial feature extraction and estimator learning. 
Recognizing human gender is important since people respond differently according to gender. In addition, A effective gender classification procedure can improve the performance of many different applications ,including person recognition and smart human-computer interfaces. In this article, we presents the problem of automatic gender identification by exploiting the physiological and aspects of the face at the same time, we explore the possibility of using head motion, mouth motion and facial appearance in a gender classification scenario.

The advantages of this system is real time face detection and tracking is possible at high detection rate, The raspberry Pi 3 processor is of low cost, Execution speed is very fast, More than one face can also be detected using this system at a time. The efficiency of system was analyzed in terms of face detection rate. The analysis revealed that the present system shows outstanding performance efficiency and can be used for face detection even from low quality images.

\section{LITERATURE REVIEW}

Face detection is used in biometrics, often as a part of (or together with) a facial recognition system. It is also used in video surveillance, human computer interface and image database management. Some recent digital cameras use face detection for autofocus [DCRP Review: Canon PowerShot S5 IS]. Face detection is also important for selecting regions of interest in images slideshows that use a pan-andscale Ken Burns effect. Face detection is gaining the interest of marketers. A webcam can be integrated into a television and detect any face that walks by. The system then estimates the race, gender, and age range of the face. Once the information is collected, a series of advertisements can be played that is specific toward the detected race/gender/age. This paper shows prototype or partial implementation of this type of work. Face detection is also being researched in the area of energy conservation [Energy Conservation]. Methodology for face recognition based on information theory approach of coding and decoding the face image is discussed in [1].

Proposed methodology is connection of two stages - Face detection using Haar Based Cascade classifier and recognition using Principle Component analysis. Various face detection and recognition methods have been evaluated [2] and also solution for image detection and recognition is proposed as an initial step for video surveillance. Implementation of face recognition using principal component analysis using 4 distance classifiers is proposed in [3]. A system that uses different distance measures for each image will perform better than a system that only uses one. The experiment show that PCA gave better results with Euclidian distance classifier and the squared Euclidian distance classifier than the City Block distance classifier, which gives better results than the squared Chebyshev distance classifier. A structural face construction and detection system is presented in [4]. The proposed system consists the different lightning, rotated facial image, skin color etc.

Lanitis et al. [5] proposed the first approach applying AAM to age estimation, which extracts craniofacial growth and skin aging during childhood and adulthood. Different classifiers (including shortest-distance classifier, quadratic function and neural networks) are compared when AAM is employed as the feature representation. The approach also differentiated between 1) age-specific estimation, which is based on the assumption that the aging process is identical for everyone; and 2) appearance-specific estimation, which follows the assumption that people who look similar tend to have similar aging processes. Subsequently, a personalized age estimation used in the specialty of aging processes is then introduced to cluster similar faces before classification. In addition, Geng et al. [6] modeled the aging process with AAM based on a sequence of age-ascending face images for the same individual. Hence, different aging models can be learnt for different persons. More specifically, Geng et al. [6], [7] introduced a personalized age estimation method that describes the long-term aging subspace of a person, called AGing pattErn Subspace (AGES).

There exists a vast literature in social and cognitive psychology describing the impressive capabilities of humans at identifying familiar faces; though, most works deal with person recognition, and only few studies are focused on gender recognition. The automatic gender recognition from human faces has been studied since the late ' $80 \mathrm{~s}$ [8][9][10], but only in the new millennium it has received significant attention from the scientific community: here we propose a short review of the latest approaches for gender recognition. Sun et al. [11] to applied principal component analysis (PCA) represent each image as a feature vector in a low dimensional space; genetic algorithms (GA) were then employed to select a subset of features form the low dimensional representation that mostly encodes the gender information. Four different classifiers were compared in this study: the Bayesian decision making, a neural network (NN), support vector machines (SVM) and a classifier based on linear discriminant analysis (LDA). The SVM achieved the best performance in the comparative experiments. Gutta et al. [12] considered a hybrid classifier for gender determination of human faces that consisted of an ensemble of radial basis functions (RBFs) and decision trees (DTs). Moghaddam et al. [13] also proposed to classify gender from facial images (of 
$21 \times 21$ pixels) using support vector machines (SVMs). They tested the SVMs by implementing different kernels and they obtained the best experimental results with the Gaussian kernel, followed by the cubic polynomial kernel.

\section{SYSTEM ARCHITECTURE}

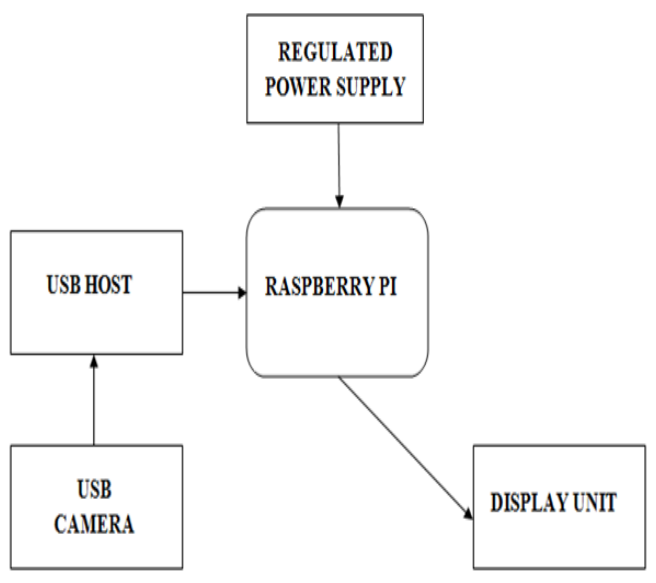

Fig.l. Basic Block Diagram

A general block diagram of the system is as shown below :

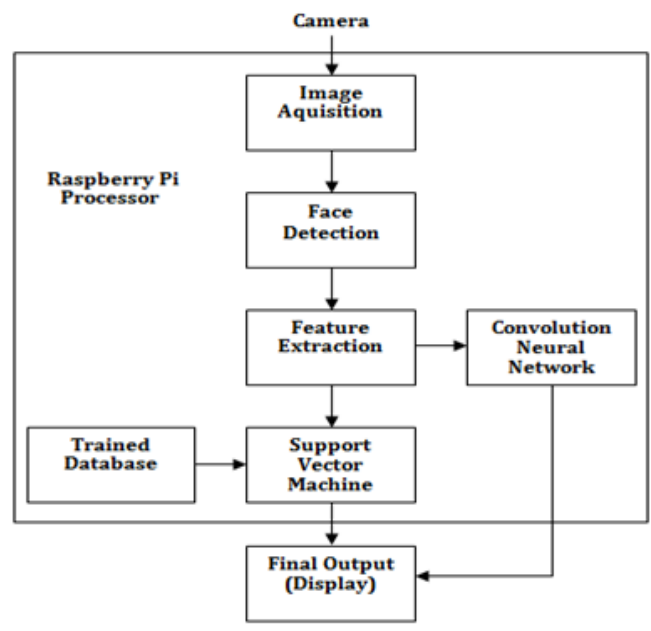

Fig.2.Block Diagram of Proposed System

\section{Working}

Firstly image is captured real time by means of the USB camera. OpenCV is used at face detection stage. OpenCV (Open Source Computer Vision) is a library of programming functions mainly designed at real-time computer vision. In simple language it is library used for Image Processing. It is mostly used to do all the operation related to Images.

Now face detection and extraction algorithm will work i.e. viola Jones algorithm which uses Haar feature based cascade classifiers algorithm for face detection. As long as a face is detected, a red bounding box is drawn on the face in the image.

Local binary pattern method which is most successful for face recognition is used for feature extraction for age estimation. After that extracted features is given to convolution neural network $(\mathrm{CNN})$ which is pre-trained model will find out whether the features extraction of an image in testing set is matching to the feature extracted from the training set and gives the estimated age according to that Histogram of oriented gradients (HOG) algorithm is used for gender and weight estimation. HOG algorithm is used for feature extraction for gender and weight estimation. Now SVM (support vector machine) will find out whether the feature extraction of an image in testing set is matching to the feature extracted from the training set. Finally output will be displayed on screen. Here Weight is classified in three classes they are under weight, normal weight and overweight.

Proposed system uses different techniques for face detection, gender estimation, age estimation and weight estimation namely :

\subsection{Viola Jones Algorithm :}

The basic principal of algorithm is to detect the faces from the given input image. Before this there were so many images processing approach but all of them were time consuming due to making the entire image to the fix size and then run the image in the detector. Opposite of this is the viola Jones algorithm were the detector is rescale and whatever the size of image would be.

The characteristics of Viola-Jones algorithm which make it a good detection algorithm are: Robust - very high detection rate (true-positive rate) \& very low false-positive rate always.

- $\quad$ Real time - For practical applications at least 2 frames per second must be processed.

- Face detection only (not recognition) - The goal is to distinguish faces from non-faces (detection is the first step in the recognition process). 


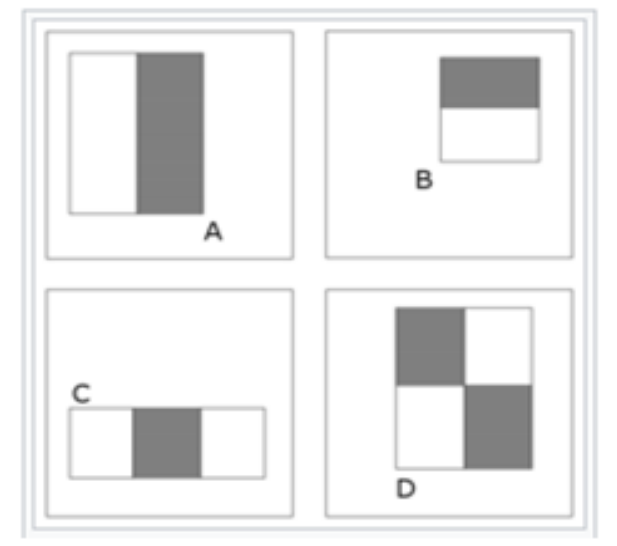

Fig.3.Feature Types used by Viola and Jones

\subsection{Histogram of oriented gradients (HOG) Algorithm :}

The histogram of oriented gradients (HOG) is a feature descriptor used in computer vision and image processing for object detection. The method counts occurrences of gradient orientation in localized portions of an image. This method is similar to that of edge orientation histograms, scaleinvariant feature transform descriptors, and shape contexts, but differs in that it is computed on a dense grid of uniformly spaced cells and uses overlapping local contrast normalization for better accuracy. It use for gender estimation procedure. The HOG descriptor maintains a few key advantages over other descriptor methods.

\subsection{Local binary patterns (LBP) :}

LBP is one of the binary patterns which is used for feature extraction. In this the face image is firstly divided into small regions from which LBP features are extracted gives the output in histogram. LBP is used because there are micro patterns which are invariant of monotonic grey scale transformation. Combining all this gives the face image. LBP is widely used in many application due to its high tolerance against object recognition texture analysis and high discriminative power.

\subsection{Support vector machines :}

In machine learning, support vector machines (SVMs) are supervised learning models with associated learning algorithms that analyze data used for classification and regression analysis. In support vector machine is used to analyze the complex data and gives the result. SVM is very useful in finding patterns which are very useful and not complex.

Classification of images can also be performed using SVMs. Experimental results show that SVMs achieve significantly higher search accuracy than traditional query refinement schemes after just three to four rounds of relevance feedback.
This is also true of image segmentation systems, including those using a modified version SVM that uses the privileged approach as suggested by Vapnik.

\subsection{Convolution Neural Network :}

In machine learning, a convolution neural network $(\mathrm{CNN})$ is a type of feed-forward artificial neural network in which the connectivity pattern between its neurons is inspired by the organization of the animal visual cortex. Individual cortical neurons respond to stimuli in a restricted region of space known as the receptive field. The receptive fields of different neurons partially overlap such that they tile the visual field. The response of an individual neuron to stimuli within its receptive field can be approximated mathematically by a convolution operation. Convolutional networks were inspired by biological processes and are variations of multilayer perceptrons designed to use minimal amounts of preprocessing. They have wide applications in image and video recognition, recommender systems and natural language processing.

\section{RESULT AND DISCUSSION}

Python codes were developed for real time face detection, tracking and gender, age and weight estimation. Photo view of project is the whole setup of project. For Real time face detection, webcam was connected to the Raspberry Pi Processor to capture the real time images of people in front of it. Output window was set to defined size and the output is displayed in it. The system is efficient to detect multiple faces and a green box was drawn across each of the detected face.

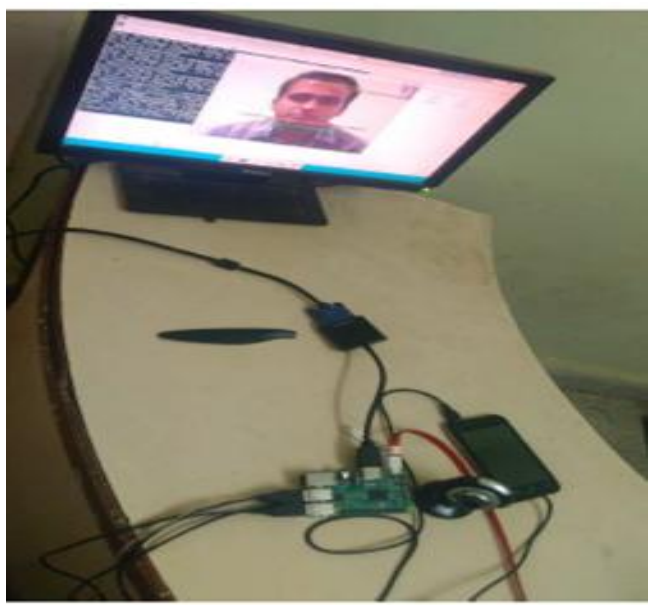

Fig-4. Photo View of Project

In output image given below face is detected, a green bounding box is drawn on the face in the image. And there is red color text on it 
displaying the gender, age and weight category of that person approx..

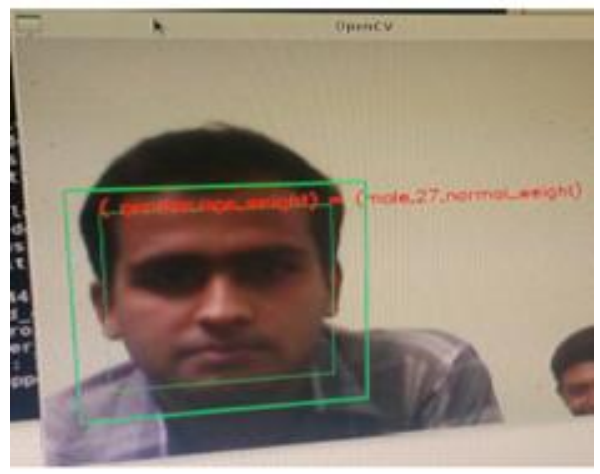

Fig.5. Output

V. CONCLUSION

A face detection, tracking and age, gender and weight estimation system using Raspberry Pi 3 model B processor was developed. The system was programmed using Python programming language. Both Real time face detection and face detection from specific images, i.e. object recognition, was carried out. The efficiency of the system was analyzed in terms of face detection rate. The analysis revealed that the present system shows excellent performance efficiency and can be used for face detection even from poor quality images.

Currently in our system we are just accessing the system from local PC system. So in future we can implement a system with video database storage for reference even which we can use single RAM for different system like servers in companies.

Currently, we focus on age estimation from faces of nearly neutral facial expressions, and evaluate our methods on the datasets without serious facial expression variations. However, expression changes could affect the age estimation results. Estimating both the age rank and facial expression intensity rank is a possible way to solve this problem.

\section{REFERENCES}

[1]. Sarala A. Dabhade \& Mrunal S. Bewoor (2012), "Real Time Face Detection and Recognition using Haar - based Cascade Classifier and Principal Component Analysis", International Journal of Computer Science and Management Research, Vol. 1, No. 1.

[2]. Faizan Ahmad, Aaima Najam \& Zeeshan Ahmed (2013), "Image-based Face Detection and Recognition: State of the Art", IJCSI International Journal of Computer Science Issues, Vol. 9, Issue. 6, No. 1.
[3]. Hussein Rady (2011), "Face Recognition using Principle Component Analysis with Different Distance Classifiers", IJCSNS International Journal of Computer Science and Network Security, Vol. 11, No. 10, Pp. 134-144.

[4]. S. Sankarakumar, Dr.A. Kumaravel \& Dr.S.R. Suresh (2013), "Face Detection through Fuzzy Grammar", International Journal of Advanced Research in Computer Science and Software Engineering, Vol. 3, No. 2.

[5]. A. Lanitis, C. J. Taylor, and T. F. Cootes, "Toward automatic simulation of aging effects on face images," IEEE Trans. Pattern Anal. Mach. Intell., vol. 24, no. 4, pp. 442-455, Apr. 2002.

[6]. X. Geng, Z.-H. Zhou, and K. Smith-Miles, "Automatic age estimation based on facial aging patterns," IEEE Trans. Pattern Anal. Mach. Intell., vol. 29, no. 12, pp. 22342240, Dec. 2007

[7]. X. Geng, Z.-H. Zhou, Y. Zhang, G. Li, and H. Dai, "Learning from facial aging patterns for automatic age estimation," in Proc. 14th Annu. ACM Int. Conf. Multimedia, 2006, pp. 307 - 316.

[8]. Golomb B.A., Lawrence D.T. and Sejnowski T.J., "Sex-Net: a neural network identifies sex from human faces", in Proceedings of Advances in neural information processing systems, pag. 572577,1990

[9]. Wiskott L., Fellous J.M., Kruger N. and Von der Malsburg C., "Face recognition and gender determination", in Proceedings on Automatic Face and Gesture Recognition, pag. 92-97, 1995.

[10]. Tamura S., Kawai H. and Mitsumoto H., "Male/female identification from 8x6 very low resolution face images by neural network", in Pattern Recognition, vol. 29, iss. 2, pag. 331-335, February 1996.

[11]. Sun Z., Bebis G., Yuan X. and Louis S.J., "Genetic feature subset selection for gender classification: a comparison study", in IEEE Proceedings on Applications of Computer Vision, pag. 165-170, 2002.

[12]. Gutta S., Huang J.R.J., Jonathon P. and Wechsler H., "Mixture of experts for classification of gender, ethnic origin, and pose of human faces", in IEEE Transactions on Neural Networks, vol. 11, iss. 4, pag. 948-960, July 2000.

[13]. Moghaddam B. and Yang M.-H., "Gender classification with support vector machines", in IEEE Proceedings on 
Automatic Face and Gesture Recognition, pag. 306-311, March 2000.

[14]. P. Viola and M. Jones. Robust Real-time Object Detection. International Journal of Computer Vision, 57(2):137-154,2002. 2, 4.

[15]. G. Yangand, T. S. Huang, "Human face detection in complex background," Pattern Recog., vol. 27, no. 1, 1994, pp. 53-63 